\title{
Best of affective computing and intelligent interaction 2013 in multimodal interactions
}

\section{Guest Editorial}

\author{
Mohammad Soleymani · Thierry Pun • Anton Nijholt
}

Received: 14 May 2014 / Accepted: 16 May 2014 / Published online: 16 July 2014

(C) OpenInterface Association 2014

The fifth biannual Humaine Association Conference on Affective Computing and Intelligent Interaction (ACII 2013) was held in Geneva, Switzerland. This conference featured the recent advancement in affective computing and relevant applications in education, entertainment and health. A number of selected papers were invited to submit an extended article to this special issue. The selection was done based on their highly favorable reviews at ACII 2013 and involvement of multimodality in their methods and applications. The review process of this special issue was performed in one cycle after which we either accepted or rejected the submissions based on the responses provided to the reviewers' comments.

This special issue features seven articles on multimodal applications or studies in affective computing. In the reminder of this editorial, we summarize the articles and their key findings in this special issue.

In "Multimodal PTSD characterization via the StartleMart game", Holmgård and colleagues [1] ran a study on physiological responses of posttraumatic stress disorder (PTSD) patients. They recorded the skin conductance (SC) and blood volume pulse (BVP) while the participants were playing a game navigating their way through a supermarket. The participants' game was interrupted by startling moments depicting combat scenes. They found significant correlations between

M. Soleymani · T. Pun

Department of Computer Science, University of Geneva,

1227 GE Carouge, Switzerland

e-mail: mohammad.soleymani@unige.ch

T. Pun

e-mail: thierry.pun@unige.ch

A. Nijholt ( $\square)$

Human Media Interaction, University of Twente,

Enschede, The Netherlands

e-mail: A.Nijholt@ewi.utwente.nl the physiological features and the stress level reported by users. These features can be used for automatic detection of the severity of PTSD.

In "Automatic nonverbal behavior indicators of depression and PTSD: the effect of gender", Stratou and colleagues [2] present how a gender specific model improves the automatic detection of depression and PTSD. In their study, the behavior of participants interacting with an artificial agent in a Wizard of Oz scenario were recorded through audiovisual channels. They identified a number of differences in the behavior associated with PTSD and depression across genders and showed how the gender-specific model outperforms the general model in automatic PTSD and depression detection from non-verbal behavior.

In "A comparative study of game mechanics and control laws for an adaptive physiological game", Parnandi and Osuna [3] presented a system that uses closed loop control systems with proportional (P) and proportional-integralderivative (PID) controllers, to adapt a car racing game to players' emotional state. Emotional state of game players was assessed from their galvanic skin response (GSR) during game play. They identified that manipulating the car speed is more effective in adapting the game to the player compared to the other parameters at hand, i.e., road visibility and vehicle steering.

In "An analysis of player affect transitions in survival horror games" by Vachiratamporn and colleagues [4] an interesting study is presented on how physiological measures can be used to predict emotional transitions in the face of horror gameplay events. During experiments various players' affective responses data were collected from gamers before and after witnessing scary events. In addition to the physiological data (heart rate, EEG), keyboard activity and self-reported emotions were also recorded. The authors discuss real-time measuring of (pre-fear) affective states such as anxiety and 
suspense, the role of different physiological measures, and the role of the pre-fear states in predicting subsequent fear levels.

In the paper "Expressive non-verbal interaction in a string quartet: an analysis through head movements" by Glowinski and colleagues [5], a study is presented where string quartet players play a piece in a standard way (concert-like) and in a variation way (where the first violinist unexpectedly adds alternate interpretations to the musical score without informing the rest of the quartet). The authors define behavioral features on the individual and group level related to head movements and orientation to measure expressivity and joint attention. Using machine learning techniques different sets of features are evaluated and the authors show how in this way they can successfully distinguish between different kinds and qualities of performances.

In "Haptic communication of dimensions of emotions using air jet based tactile stimulation", Tsalamlal and colleagues [6] investigate the use of mobile air jet to apply a non-intrusive tactile stimulation to different and possibly large areas of the body. They assessed the affective reactions to such air jets along the valence, arousal and dominance dimensions. Amongst the possible applications of such stimulation could be haptic feedback from virtual agents to communicate affect during interaction.

In "Social support agents for older adults: longitudinal affective computing in the home", Ring and colleagues [7] describe a conversational agent-based system designed to provide longitudinal social support to isolated older adults. A pilot study was conducted in which the agent was placed in the homes of 14 older adults for a week. Results indicate high levels of acceptance and satisfaction of the system, as well as the need for proactively drawing the elders into interaction.
Affective component is a prominent component of any human-computer interfaces and as presented in the articles of this special issue have broad applications from health and entertainment to art. We believe that this special issue presents important and timely contributions to the field of affective computing.

Acknowledgments The guest editors of this special issue would like to thank all the anonymous reviewers for their valuable comments and the feedback they provided to the extended manuscripts. We also thank the authors to accept our invitation and for their contribution to the advancement of the state of the art in this field.

\section{References}

1. Holmgård C, Yannakakis GN, Martínez HP, Karstoft KI, Andersen HS (2014) Multimodal PTSD characterization via the startlemart game. J Multimodal User Interf . doi:10.1007/s12193-014-0160-5

2. Stratou G, Scherer S, Gratch J, Morency LP (2014) Automatic nonverbal behavior indicators of depression and PTSD: the effect of gender. J Multimodal User Interf. doi:10.1007/s12193-014-0161-4

3. Parnandi A, Osuna RG (2014) A comparative study of game mechanics and control laws for an adaptive physiological game. J Multimodal User Interf. doi:10.1007/s12193-014-0159-y

4. Vachiratamporn V, Legaspi R, Moriyama K, Ichi Fukui K, Numao M (2014) An analysis of player affect transitions in survival horror games. J Multimodal User Interf. doi:10.1007/s12193-014-0153-4

5. Glowinski D, Dardard F, Gnecco G, Piana S, Camurri A (2014) Expressive non-verbal interaction in a string quartet: an analysis through head movements. J Multimodal User Interf. doi:10.1007/ s12193-014-0154-3

6. Tsalamlal MY, Ouarti N, Martin JC, Ammi M (2014) Haptic communication of dimensions of emotions using air jet based tactile stimulation. J Multimodal User Interf. doi:10.1007/s12193-014-0162-3

7. Ring L, Shi L, Totzke K, Bickmore T (2014) Social support agents for older adults: longitudinal affective computing in the home. $\mathrm{J}$ Multimodal User Interf. doi:10.1007/s12193-014-0157-0 